\title{
Serviceorientiertes Supply Chain Event Management (SCEM) - Nutzen und Architektur für globale Lieferketten am Beispiel der Modeindustrie
}
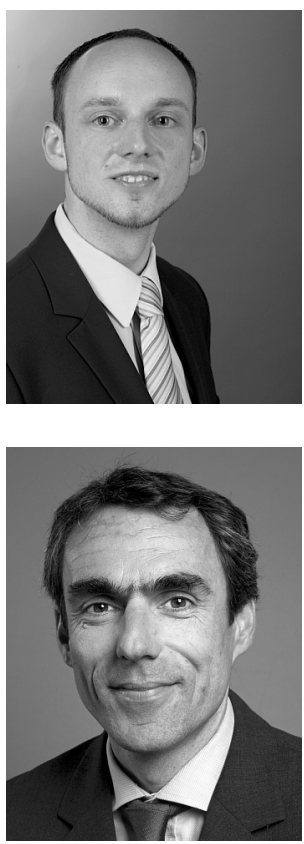

\section{Ralph Tröger und Rainer Alt}

Supply Chain Event Management, SCEM, Serviceorientierte Architektur, SOA, Services, Serviceorientierung, Modeindustrie

Supply Chain Event Management, SCEM, service-oriented architecture, SOA, services, service-orientation, fashion industry

Serviceorientierte Architekturen (SOA) erfahren in der betrieblichen Praxis zunehmende Verbreitung und tragen zu höherer Effizienz von Geschäftsprozessen bei. Supply Chain Event Management (SCEM) ist ein Konzept zur Erreichung unternehmensübergreifender Transparenz, das eine rechtzeitige Reaktion auf kritische Ausnahmeereignisse („Events“) ermöglicht. Für das Management globaler Supply Chains können beide Themengebiete einen Beitrag leisten, jedoch fehlt bislang eine konzeptionelle Verbindung der bis dato separat untersuchten Ansätze. Hier setzt dieser Beitrag an und systematisiert ausgehend von den Nutzenpotenzialen des SCEM mögliche Ansatzpunkte der Serviceorientierung. Die Ausgestaltung einer serviceorientierten SCEM-Architektur illustriert abschliessend ein Beispiel aus der Modeindustrie.

Service-oriented architectures (SOA) are spreading in many organisations and aim at enhancing efficiency of business processes. Supply Chain Event Management (SCEM) may be characterised as an approach to achieve inter-organisational visibility enabling companies to react to critical exceptions (,events") in time. To date, though relevant for the management of global supply chains, SOA and SCEM have received only little attention in combination. This research consolidates the potential benefits of SCEM and aims to elaborate their implications for service-orientation. A possible service-oriented architecture for SCEM is finally designed using the example of the fashion industry.

\section{Einleitung}

\subsection{Motivation}

Verantwortliche im Supply Chain Management (SCM) verwenden etwa 60\% ihrer Arbeitszeit auf die Behebung der Auswirkungen von Störungen (Kapazitätsengpässe, Lieferschwierigkeiten, Produktionsverzögerungen, etc.) in der Lieferkette (engl.: Supply Chain) (Speyerer/Zeller 2004, $1 \mathrm{f}$.). Aus den beobachtbaren branchenübergreifenden Trends in 
den Beschaffungsstrategien (Internationalisierung, kürzere Produktlebenszyklen, etc.) lässt sich schliessen, dass sowohl die Komplexität des SCM als auch die Störanfälligkeit globaler Supply Chains weiter zunehmen werden (Straube et al. 2007, 12 ff.). Ein Ansatz zur Bewältigung dieser wachsenden Komplexität bildet das SCEM, das wiederum auf dem Ansatz des Management by Exception (MbE) beruht. MbE besagt im Wesentlichen, die Aufmerksamkeit von Verantwortungsträgern nur im Bedarfsfall zu beanspruchen (Bittel 1964, 5).

Bedeutender als die durch diese kritischen Ausnahmeereignisse (Events) entstehende Arbeitslast im operativen Tagesgeschäft (z. B. zusätzlicher Planungs- und Koordinationsaufwand) sind jedoch deren mittel- und langfristige Folgen für Unternehmen. Nach Schulte (2009, 7 ff.) sind die zentralen Ziele der Logistik, die mehrere Autoren synonym zum SCM verwenden (Werner 2008, 12), die Logistikleistung (u. a. Lieferzeit, -treue, Flexibilität) sowie die Logistikkosten (bspw. Steuerungs-, System-, Transport-, Umschlag-, Lagerungskosten). Da beide durch das Auftreten von Ausnahmeereignissen bzw. Events gefährdet sind, bietet SCEM das Potenzial, Logistikkosten und -leistung wieder positiv zu beeinflussen (siehe Müller et al. 2009). Für internationale Supply Chains verspricht das Konzept somit unmittelbare Auswirkungen auf die Wettbewerbsfähigkeit.

Informationssysteme (IS) sind eine zentrale Voraussetzung zur Realisierung von SCEMLösungen (bspw. Otto 2003, 4 ff.). Obgleich mehrere Beiträge (Nissen 2002; Heusler et al. 2006; Ijioui et al. 2007, etc.) die Nutzenpotenziale von SCEM-Systemen untersuchen, scheint nach Sichtung der Literatur ein systematischer Überblick über die Nutzenpotenziale des SCEM in der Praxis zu fehlen. Ein weiteres Defizit stellt die Anwendung innovativer informationstechnologischer Architekturkonzepte dar. Hier schaffen serviceorientierte Architekturen neue Impulse bezüglich einer höheren Effizienz und Flexibilität von IS ( $\mathrm{Erl}$ 2008, 38). Jedoch hat sich bislang noch kein Beitrag mit der Anwendung von SOA im Umfeld von SCEM befasst.

Vor diesem Hintergrund fasst dieser Beitrag nach einer knappen Klärung der Themengebiete SCEM und SOA die in der Literatur diskutierten Nutzendimensionen des SCEM zusammen und identifiziert darauf aufbauend die möglichen Ansatzpunkte für den SOA-Einsatz im SCEM. Den Beitrag schliesst das Beispiel einer serviceorientierten SCEM-Systemarchitektur aus Sicht eines fokalen Unternehmens in einer globalen Supply Chain der Modeindustrie $\mathrm{ab}$.

\subsection{Supply Chain Event Management}

SCEM ist ein Konzept zur Erreichung unternehmensübergreifender Visibilität logistischer Prozesse, das eine rechtzeitige Reaktion auf kritische Ausnahmeereignisse („Events“) erlaubt (vgl. u. a. Nissen 2002; Heusler et al. 2006; Bretzke et al. 2002 sowie Otto 2003). SCEM ist dabei abzugrenzen vom reinen Tracking \& Tracing, da es Soll-Ist-Abweichungen in Event-Benachrichtigungen übersetzen kann und SCEM-Systeme überdies die Fähigkeit besitzen, auf Basis „... hinterlegter Regeln Handlungsvorschläge [zu] generieren oder - im Extremfall - das Problem selbstständig [zu] lösen.“ (Bretzke et al. 2002, 2). Als Grundfunktionalitäten des SCEM gelten Überwachen, Melden, Simulieren, Steuern und Messen (siehe Abbildung 1).

Den möglichen Nutzen dieser Funktionalitäten illustrieren zahlreiche Studien (Bretzke et al. 2002; Ijioui et al. 2007; Tröger et al. 2008). Dazu zählen ein reduzierter Kontrollaufwand für die Prozessverantwortlichen, eine Gesamtdurchlaufzeitreduzierung, eine höhere 


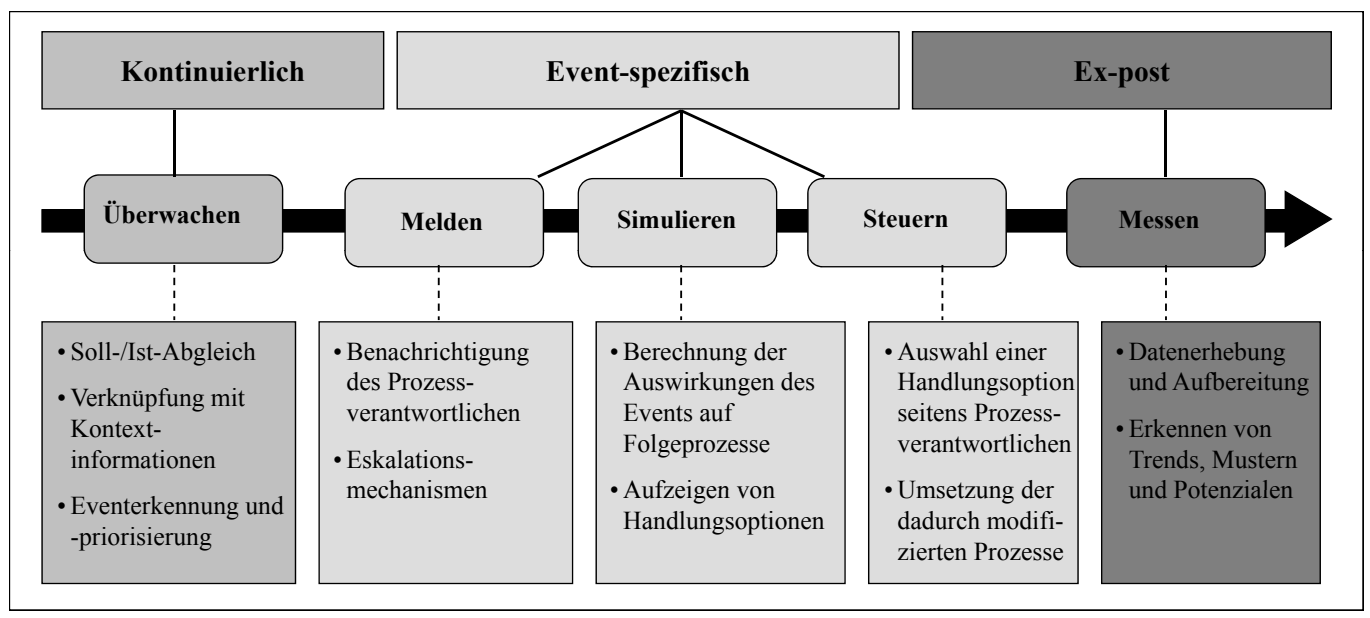

Abbildung 1: Grundfunktionalitäten des Supply Chain Event Management

Prozesstransparenz, eine verbesserte Liefertermintreue, die Minimierung von Fehlerfortpflanzungen, Kapazitätserhöhungen, die Entlastung von Routineaufgaben sowie die Aufdeckung von Schwachstellen in der Lieferkette zur mittel- und langfristigen Optimierung der Supply Chain.

Als mehrstufig räumlich und institutionell verteilte Systeme beinhalten logistische Ketten immer auch Risiken. Dazu zählen Lieferkettenrisiken wie Abweichungen (z. B. Bestellmengenänderung), Störungen (bspw. durch Maschinenausfall verursachte Produktionsverzögerung) und Katastrophen (Terroranschlag o. ä.) (Gaonkar/Viswanadham 2004). Letztere sind durch Unternehmen in den meisten Fällen nicht steuerbar, da weder deren Ursachen noch Wirkungen in deren Gestaltungs- bzw. Einflussbereich liegen. Weiterhin unterscheiden Gaonkar/Viswanadham (2004) drei Planungsebenen im SCM: operativ (bspw. Umplanung von Transporten), taktisch (Erarbeitung von Notfallplänen u. ä.) und strategisch (Partnerauswahl, etc.). SCEM gilt hierbei als „... Brücke zwischen der mittel- bis langfristig ausgerichteten Planung (...) und der operativen Ausführung dieser Pläne (...)“ (Heusler et al. 2006, 19).

Da SCEM gemäss seiner Funktionalitäten Unternehmen sowohl bei der reaktiven Behebung von Ausnahmeereignissen (insbesondere durch Überwachen/Melden/Simulieren/Steuern) als auch bei der präventiven Optimierung der Supply Chain (hauptsächlich durch Messen) unterstützt, kann SCEM auf operativer Ebene reaktiv sowie auf taktischer/strategischer Ebene präventiv zum Risikomanagement von Supply Chains beitragen (vgl. hierzu Abbildung 2).

\subsection{Serviceorientierung und serviceorientierte Architektur}

SOA folgt dem Designparadigma der Serviceorientierung, das als Service Science gegenwärtig eine zunehmende Konkretisierung erfährt. In einer SOA bezeichnet ein „Service“ den elementarsten Bestandteil von serviceorientierten Lösungen (Erl 2008, 39). Als unternehmerische Ziele einer SOA gelten die Steigerung unternehmerischer Effizienz, Agilität und Produktivität ( $E r l 2008,38)$. Eine SOA umfasst typischerweise drei Elemente: 


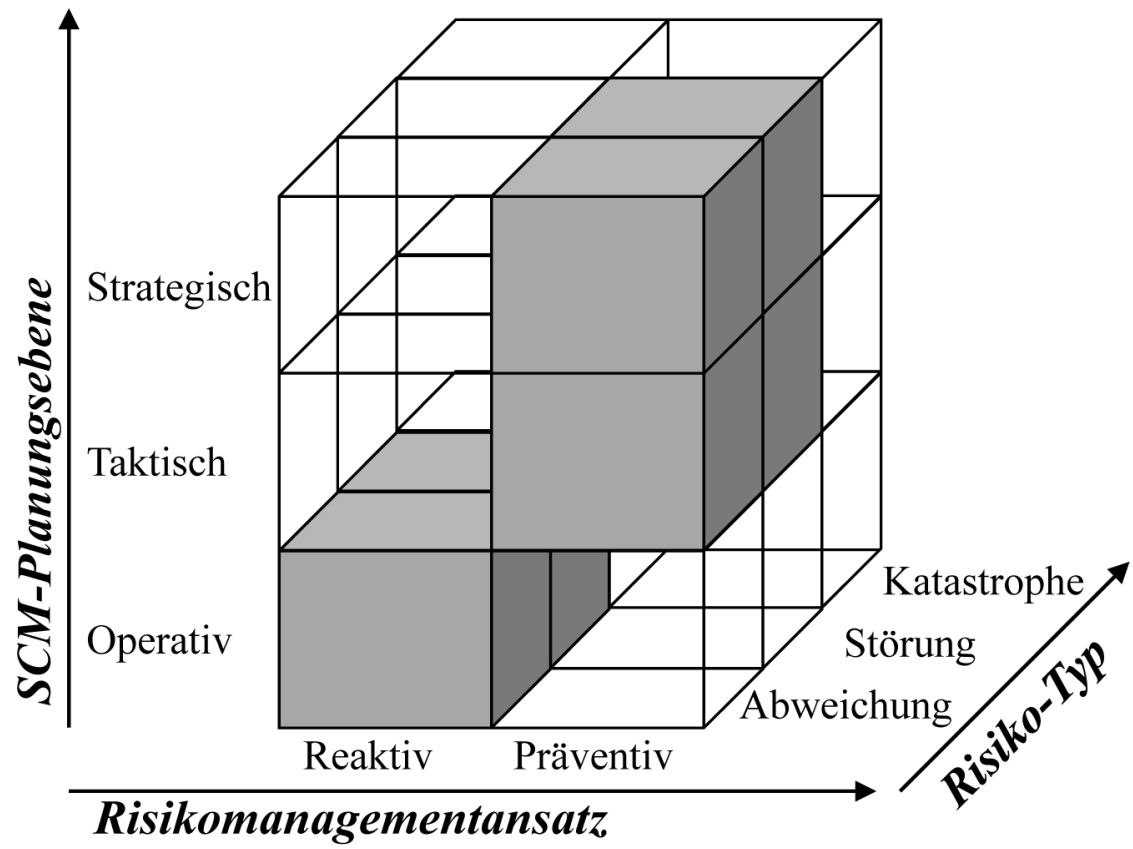

Abbildung 2: Risikomanagement globaler Lieferketten mit SCEM

Services bezeichnen wohldefinierte, unabhängige und wieder verwendbare Module, die spezifische, in Applikationen gekapselte Funktionalitäten über eine standardisierte Schnittstelle bereitstellen. Services lassen sich nach ihrem Grad des fachlichen Bezuges und ihrer Granularität unterscheiden, z. B. in Geschäfts-, Applikations- und IT-Services (Kohlmann/Alt 2009 a, 204). Aus technischer Sicht existieren die sog. Web Services Standards (Papazoglou/Van den Heuvel 2007, 390), wobei die „Web Service Description Language“ zur Servicebeschreibung, das „Simple Object Access Protocol“ zur Nachrichtenübertragung und der „Universal Description, Discovery and Integration“(UDDI) als Verzeichnisstandard dient.

Service-Verzeichnisse enthalten als wichtiges komplexitätsreduzierendes Architekturelement in einer SOA die Servicebeschreibung, die Schnittstellenspezifikation sowie das hinterlegte Preismodell der Services (Kohlmann/Alt 2009 b, 105). Sie bieten Serviceanbietern die Möglichkeit, Services zu registrieren, sodass Kunden diese finden und nutzen können. Im Sinne eines Service-Marktplatzes bringen sie Anbieter und Nachfrager von Services zusammen (Legner 2009, 70). Jüngere Entwicklungen wie etwa die „Universal Service Description Language“ (USDL) verbessern neben UDDI mit dem Ziel einer weitergehenden Automatisierung die formale Beschreibung der Services.

Ein Enterprise Service Bus (ESB) schliesslich fungiert als Integrationsplattform einer SOA und kann über standardisierte Adapter und Schnittstellen die Interoperabilität zwischen Applikationen und anderen Komponenten herstellen (Papazoglou/Van den Heuvel 2007, 390). Ferner unterstützt ein ESB die Implementierung und das Management der durch Service-Verzeichnisse bereitgestellten Dienste. Bekannte ESB-Anbieter sind u. a. Sun 
Microsystems („Open ESB“), IBM („WebSphere Enterprise Service Bus“), Microsoft („Biztalk Server“) und Oracle („Oracle ESB“).

\subsection{Forschungsfragen und Forschungsmethode}

Obgleich SOA den Lösungen von zahlreichen Standardsoftware-Anbietern und auch Anwendern zugrunde liegt, steht die Anwendung für die Domäne des SCEM noch aus. Da SCEM-Systeme u. a. durch eine inhärent hohe Anzahl potenzieller Schnittstellen, unternehmens- und plattformübergreifende Integration sowie durch hohe Anforderungen bzgl. Flexibilität geprägt sind, scheinen SCEM-Systeme eine vielversprechende Anwendung im SOA-Kontext zu sein. Hieraus ergeben sich zwei Forschungsfragen, die eine Literaturanalyse beantworten soll:

(1) Inwieweit kann SOA zum Nutzen von SCEM-Systemen beitragen?

(2) Wie kann ein serviceorientiertes SCEM in der Modeindustrie gestaltet sein?

\section{SOA als Nutzentreiber von SCEM}

\subsection{SCEM-Nutzenpotenziale im Überblick}

Eine konsolidierte Sicht der bisherigen Forschungsergebnisse zu SCEM-Nutzenpotenzialen (s. Bretzke et al. (2002); Nissen (2002); Stölzle (2004); Bodendorf/Zimmermann (2005); Heusler et al. (2006); Ijioui et al. (2007); Tröger et al. (2008); Müller et al. (2009) sowie Tröger/Alt (2010)) vermittelt Tabelle 1. Im Vordergrund stehen einerseits die Nutzenkategorie (quantitativ vs. qualitativ) und andererseits die SCM-Nutzenebenen, die sich in Anlehnung an Bose/Pal $(2005,103$ f.) in strategisch, taktisch und operativ unterscheiden lassen.

\subsection{Determinanten des SCEM-Nutzens}

Um die seitens der Unternehmen bzw. Lieferketten gesetzten Ziele zu erreichen, ist eine optimale Ausrichtung von Strategie, Prozessen und IT erforderlich. Folglich lässt sich der Nutzen des SCEM nur dann in vollem Umfang realisieren, wenn die jeweiligen Informationssysteme hinreichend gut auf die Anforderungen der SCEM-Prozesse abgestimmt sind. Aus den zusammengetragenen Nutzenpotenzialen lassen sich in diesem Sinne die folgenden Nutzendeterminanten ableiten:

(a) Hoher unternehmensinterner und -übergreifender IT-Integrationsgrad: Insbesondere zur frühzeitigen Information der Prozessverantwortlichen über kritische Plan-/Ist-Abweichungen sowie als Datenbasis zur Generierung von Handlungsvorschlägen benötigen SCEM-Systeme jederzeit Zugriff sowohl auf Plan-Daten (z. B. vereinbarte Liefertermine und -mengen) als auch auf Ist-Daten (u. a. Tracking \& Tracing (T\&T), Verkehrsinformationen, Frachttarife, Lagerbestände, etc.) der Supply Chain. Je höher der Integrationsgrad zu unternehmenseigenen Applikationen (bspw. Enterprise Resource Planning (ERP), EMail, Data Warehouse, Human Resource Management, Warenwirtschaft, etc.) und fremden Anwendungssystemen (Transportmanagement, ERP, Electronic Product Code Information Services (EPCIS) Repositories, T\&T, Wetter-/Nachrichten-/Verkehrsportale, etc.), desto effektiver kann ein SCEM funktionieren.

(b) Bedarfsgerechte Informationsversorgung: Ein SCEM-System soll nur diejenigen Informationen weitergeben, „... deren Kenntnis für einen spezifischen Empfänger wesentlich 
ist und einen dementsprechenden Handlungsimpuls beinhalten" (Heusler et al. 2006, 20 f.). SCEM-Systeme übernehmen in diesem Zusammenhang die Funktion eines Filters, die den Prozessverantwortlichen die manuelle Suche nach kritischen Abweichungen erspart (Bretzke et al. 2002, 2).

\begin{tabular}{|c|c|c|}
\hline \multirow[t]{2}{*}{ Nutzenebene } & \multicolumn{2}{|l|}{ Nutzenkategorie } \\
\hline & Quantitativ & Qualitativ \\
\hline \multirow[t]{2}{*}{ Operativ } & \multirow{2}{*}{$\begin{array}{l}\text { Höhere Mitarbeiterproduktivität durch Pro- } \\
\text { zessautomatisierung und -unterstützung (z. } \\
\text { B. Systeme auf Plan-/Ist-Abweichungen kon- } \\
\text { trollieren, Events klassifizieren/priorisieren, } \\
\text { Prozessverantwortliche/Netzwerkpartner be- } \\
\text { nachrichtigen, Kontextinformationen be- } \\
\text { schaffen, Meldungen eskalieren, Informatio- } \\
\text { nen transformieren, Eventauswirkungen ab- } \\
\text { schätzen, Handlungsalternativen identifizie- } \\
\text { ren, Informationen zu Handlungsalternati- } \\
\text { ven einholen, Optionen bewerten, Bestell-/ } \\
\text { Produktions-/Transport-/Kundenaufträge } \\
\text { buchen/modifizieren/stornieren, Daten selek- } \\
\text { tieren/extrahieren/transformieren/dokumen- } \\
\text { tieren, Analysen durchführen, Kennzahlen } \\
\text { bilden und aktualisieren, etc.) }\end{array}$} & $\begin{array}{l}\text { Informationsflutreduzierung } \\
\text { (z. B. durch adressatenge- } \\
\text { rechte Benachrichtigungen, } \\
\text { Priorisierung von Events, } \\
\text { Alarmierung nur bei kriti- } \\
\text { schen Plan-/Ist-Abweichun- } \\
\text { gen, etc.) und Reduzierung } \\
\text { der Prozesskomplexität }\end{array}$ \\
\hline & & $\begin{array}{l}\text { Entscheidungsunterstützung } \\
\text { (z. B. durch Kalkulation der } \\
\text { Eventauswirkungen, Zusam- } \\
\text { menstellung der Kontextin- } \\
\text { formationen, Generierung } \\
\text { von Handlungsvorschlägen } \\
\text { zu Neu- bzw. Umplanung } \\
\text { von Logistikprozessen, etc.) }\end{array}$ \\
\hline \multirow[t]{2}{*}{ Taktisch } & \multirow{2}{*}{$\begin{array}{l}\text { Senkung der Logistik-/SCM-Kosten (z. B. } \\
\text { durch Vermeidung bzw. Reduzierung von } \\
\text { Konventionalstrafen/Nachlieferungen/ } \\
\text { Sonderfahrten/Reklamationen/Retouren/ } \\
\text { Fehlerfortpflanzungen, Durchlaufzeitredu- } \\
\text { zierungen, bessere Kapazitätsauslastung, } \\
\text { niedrigere Bestände, bessere Datenbasis zur } \\
\text { Klärung von Verschuldungsfragen, etc.) }\end{array}$} & $\begin{array}{l}\text { Steigerung der Logistikleis- } \\
\text { tung (z. B. durch höhere Ter- } \\
\text { mintreue, weniger Fehl- bzw. } \\
\text { Falschlieferungen, beschleu- } \\
\text { nigte Prozesse, effektivere } \\
\text { Kundenbetreuung, usw.) }\end{array}$ \\
\hline & & $\begin{array}{l}\text { Erhöhung der Prozesstrans- } \\
\text { parenz (z. B. durch aktuelle- } \\
\text { re/genauere Informationen) }\end{array}$ \\
\hline \multirow[t]{2}{*}{ Strategisch } & $\begin{array}{l}\text { Steigerung des Unternehmenswertes/Unter- } \\
\text { nehmensgewinns durch geringere Kosten in } \\
\text { Logistik/SCM sowie Steigerung des Umsat- } \\
\text { zes durch Vermeidung von Umsatzverlusten } \\
\text { (z. B. durch Vermeidung von Out-of-Stock- } \\
\text { Situationen) }\end{array}$ & $\begin{array}{l}\text { Entscheidungsgrundlage zur } \\
\text { Optimierung der Logistik- } \\
\text { strukturen und Supply Chain } \\
\text { Prozessen durch Aufdeckung } \\
\text { systematischer Schwachstel- } \\
\text { len wie z. B. Kapazitätseng- } \\
\text { pässen an bestimmten Liefer- } \\
\text { kettenabschnitten }\end{array}$ \\
\hline & $\begin{array}{l}\text { Steigerung des Unternehmenswertes/Unter- } \\
\text { nehmensgewinns durch geringere Kosten im } \\
\text { Customer Relationship Management durch } \\
\text { Steigerung der Kundenzufriedenheit, mithin } \\
\text { der Kundenbindung (Kosten zur Kundenbe- } \\
\text { ziehungspflege geringer als Kosten zur Ak- } \\
\text { quise von Neukunden) }\end{array}$ & $\begin{array}{l}\text { Zeitgewinn bei Entschei- } \\
\text { dungsprozessen (Informati- } \\
\text { onsvorteil ermöglicht früh- } \\
\text { zeitige Anpassung von Mass- } \\
\text { nahmen an geänderte Rah- } \\
\text { menbedingungen) }\end{array}$ \\
\hline
\end{tabular}

Tabelle 1: Systematisierung von SCEM-Nutzenpotenzialen 
(c) Hobe Prozessautomatisierung und -unterstützung: In engem Zusammenhang mit dem vorherigen Punkt zeichnen sich SCEM-Systeme dadurch aus, auf „... Grundlage hinterlegter Regeln Handlungsvorschläge [zu] generieren oder (...) das Problem selbstständig [zu] lösen“ (Bretzke et al. 2002, 2). Insbesondere hinsichtlich der SCEM-Funktionen ,Simulieren' und ,Steuern' sind bedeutende Arbeitsentlastungspotenziale möglich, da hier der Aufgabenschwerpunkt von Supply Chain Managern liegt (u. a. Abschätzung der Auswirkungen von Events; Datenbeschaffung für verschiedene Neu- bzw. Umplanungsalternativen von Supply Chain Prozessen; Umsetzung der Neu-/Umplanung wie bspw. Abstimmung mit Wertschöpfungspartnern, Dateneingaben, etc.).

(d) Hohe Flexibilität bei Konfiguration: Eventtypen, im $\mathrm{SC}(\mathrm{E}) \mathrm{M}$ zu unterstützende Geschäftsprozesse, Anzahl und Identität der Wertschöpfungspartner (Lieferanten, Produzenten, Logistik- bzw. Transportdienstleister), Zuständigkeiten und IT-Systeme sind für jede Supply Chain individuell und unterliegen einem ständigen Wandel. Ein SCEM-System muss daher leicht an die sich ändernden Erfordernisse und Rahmenbedingungen anzupassen sein.

Durch die hohe Flexibilität, die vergleichsweise leichte Anwendungssystemintegration und die Möglichkeiten der Service-Orchestrierung unterstützt eine SOA insbesondere die Nutzendeterminanten (a), (c) und (d). Letztere sind vor dem Hintergrund einer guten Wettbewerbsfähigkeit gerade für international aufgestellte Lieferketten mit zumeist zahlreichen/häufig wechselnden Wertschöpfungspartnern bzw. einer hohen Systemheterogenität von hoher Bedeutung. Der nächste Abschnitt untersucht dazu, inwieweit die in der wissenschaftlichen Diskussion bislang identifizierten SOA-Potenziale den Nutzen einer serviceorientierten SCEM-Systemarchitektur weiter verstärken können.

\subsection{SCEM-relevante SOA-Nutzeffekte}

Legner/Heutschi (2007, 1649 ff.) zeigen in ihrer Studie früher SOA-Implementierungen in der betrieblichen Praxis sowohl kosten-, zeit- als auch qualitätsbezogene Nutzeffekte auf. Für unternehmensübergreifende Anwendungssysteme wie SCEM sind davon insbesondere geringere Systemintegrations-, Entwicklungs- und Betriebskosten (durch Schnittstellenstandardisierung, Wiederverwendung und Redundanzreduktion), schnellere Prozessanpassungen (durch Trennung von Geschäfts- von Prozesslogik) sowie integrierte Prozesse und Investitionsschutz für bestehende Systeme (durch plattformübergreifende Nutzung verschiedener Funktionen) bedeutsam.

Weitere SCEM-relevante Potenziale lassen sich aus der modellgetriebenen SOA-Nutzenanalyse von Müller et al. $(2010,169)$ ableiten, die - ausgehend von den drei SOA-Designprinzipien Modularität, Orientierung an Standards und loser Kopplung - u. a. höhere Verfügbarkeit von Informationen, effizientere Geschäftsprozesse sowie verbesserte unternehmensübergreifende Koordination und Kommunikation nennen. Basierend auf diesen Vorarbeiten führt Tabelle 2 jene SOA-Nutzeffekte auf, die zusätzlichen Mehrwert für SCEMRealisierungen versprechen.

In Beantwortung von Forschungsfrage 1 lässt sich somit schlussfolgern, dass durch die inhärente Unterstützung der Nutzendeterminanten von SCEM-Systemen einerseits sowie durch die zusätzlichen Nutzeffekte auf Kosten-, Zeit- und Qualitätsebene andererseits eine serviceorientierte Architekturgestaltung von SCEM-Systemen eine vielversprechende Option darstellt. Eine mögliche Ausgestaltung illustriert im folgenden Kapitel das Beispiel der Modeindustrie. 


\begin{tabular}{|c|c|}
\hline SOA-Nutzeffekte auf: & ... ergänzen SCEM-Nutzenpotenziale durch: \\
\hline (a) Kostenebene & $\begin{array}{l}\text { - Geringere Systemintegrationskosten (durch Schnittstellenstandar- } \\
\text { disierung) } \\
\text { - Geringere Betriebskosten (durch Reduktion von Redundanzen - } \\
\text { so werden zahlreiche der von Tröger/Alt (2010) identifizierten } \\
\text { SCEM-relevanten Funktionalitäten auch von anderen Applikatio- } \\
\text { nen benötigt) } \\
\text { - Geringere Entwicklungskosten (insb. durch Wiederverwendung } \\
\text { gekapselter Funktionalitäten, siehe vorherigen Punkt) } \\
\text { - Potenzielle Mehreinnahmen durch Bereitstellung von Services } \\
\text { (mögliches Geschäftsmodell für Produzenten, Logistik- und } \\
\text { Transportdienstleister) }\end{array}$ \\
\hline (b) Zeitebene & $\begin{array}{l}\text { - Flexibilität bei Konfiguration (z. B. Eventmuster/SCEM-relevante } \\
\text { Prozesse) } \\
\text { - Verkürzung von (SCEM-)Projektdurchlaufzeiten } \\
\text { - Schnellere Anpassungen (z. B. bei wechselnden Partnern bzw. sich } \\
\text { ändernden Geschäftsobjekten) }\end{array}$ \\
\hline (c) Qualitätsebene & $\begin{array}{l}\text { - Vereinfachte Kommunikation zwischen IT- und (SCM- bzw. Lo- } \\
\text { gistik-)Fachbereich } \\
\text { - Investitionsschutz für bestehende Systeme } \\
\text { - Höhere Informationsverfügbarkeit (Vorhandensein zahlreicher } \\
\text { standardisierter SCEM-relevanter Informationsdienste wie z. B. } \\
\text { T\&T-Services wie ,TrackIT' von DHL, etc.) }\end{array}$ \\
\hline
\end{tabular}

Tabelle 2: Zusätzliche SCEM-Nutzeffekte durch SOA

\section{Serviceorientierte SCEM-Systemarchitektur}

\subsection{Schwachpunkte bisheriger SCEM-Architekturen}

Bestehende SCEM-Architekturkonzepte in der Literatur weisen Schwächen auf, die sich gleichzeitig als Potenziale für den Einsatz von SOA interpretieren lassen. Dazu zählen (vgl. Tröger/Alt 2010; Wieser/Lauterbach 2001; Nissen 2002; Otto 2003; Speyerer/Zeller 2004; Bodendorf/Zimmermann 2005; Kurbel/Schreber 2005 sowie Tribowski et al. 2009):

(a) keine vollständige Abdeckung SCEM-relevanter Funktionalitäten (insbesondere zu den Funktionalitäten ,Simulieren' und ,Steuern'),

(b) zum Teil zu anbieterspezifische Betrachtung (3 von 7 Konzepten bezogen sich auf die SAP-Produktfamilie) sowie

(c) teilweise zu fokussierte Betrachtungsweise (4 von 7 Ansätzen analysieren SCEM nur aus einer bestimmten technologischen Perspektive wie Agenten, EPCIS, etc.).

Grundsätzlich lassen sich durch die Kombination verteilter (Web-)Services alle SCEM-relevanten Funktionalitäten abdecken (Tröger/Alt 2010), womit ein serviceorientierter Ansatz den erstgenannten Schwachpunkt (a) ausräumen kann. Vor dem Hintergrund der SOA-inhärenten Eigenschaften der Anbieter- und Technologieunabhängigkeit gilt dies auch für die Punkte (b) und (c). 


\subsection{Identifizierung SCEM-relevanter Services}

Das Ziel der Service Science besteht darin, „... die Entwicklung innovativer Services durch geeignete Methoden und formale Modelle zu unterstützen und das Management von Services zu verbessern (...) “ (Bubl et al. 2008, 60). Als Grundlage zur Identifizierung der für ein SCEM notwendigen (Business) Services dient das auf dem Business Engineering-Ansatz (Österle 1995) aufbauende Architekturkonzept von Kohlmann/Alt (2009 a sowie 2009 b) (siehe Abbildung 3). Dieses verfolgt einen hybriden Ansatz, der eine geschäftsorientierte (Top-down) mit einer technologiegetriebenen (Bottom-up) Service-Identifikation verbindet. Zusammengefasst geht das dominierende Top-down von den jeweiligen Geschäftsmodellen bzw. -prozessen aus, während Bottom-up auf die Service-Modellierung existierender Applikationen und deren Funktionalitäten abzielt (Koblmann/Alt 2009 a, 204). Der nachfolgende Ansatz modifiziert dieses Konzept in drei Punkten:

(1) Analyse der Lieferkette einschliesslich der Geschäftsmodelle, Strukturen, Rollen, Hauptprozesse und Abhängigkeiten. (vgl. Abbildung 3: „Strategie“)

(2) Bestimmung der benötigten SCEM-relevanten Funktionalitäten und korrespondierenden Services auf Basis der potenziell vorkommenden Events und der im Rahmen des SCEM-Funktionsumfangs (Überwachen, Melden, Simulieren, Steuern, Messen) zu unterstützenden Geschäftsprozesse. (vgl. Abbildung 3: „Prozesse“ und „Services“)

(3) Bestimmung potenzieller Service-Provider (bspw. unternehmensinterne Applikationen, die die unter Schritt (2) identifizierten Funktionalitäten als gekapselte Funktion bereitstellen können bzw. externe (Web-)Service-Provider). (vgl. Abbildung 3: „Informationssysteme“ und „Prozesse“)

Auf Basis dieser Systematik haben Tröger/Alt (2010) im Rahmen einer Fallstudie in der Modeindustrie anhand der zehn häufigsten kritischen Events die SCEM-Services auf Geschäftsebene („Business Services“) identifiziert. Dies ist nachfolgend für jede der fünf SCEM-Grundfunktionalitäten anhand des Events ,Produktionsverzögerung` exemplarisch dargestellt:

Überwachen: Zur Detektion einer Verzögerung im Produktionsprozess benötigt ein Unternehmen von den Produzenten im Idealfall Prozess-Tracking- bzw. EPCIS-Daten. Beide Funktionalitäten (Abfrage des Produktionsstatus über eine Auftragsplattform sowie Abfrage eines EPCIS-Repositories) kann ein Produzent kapseln und dem Modeunternehmen als Service zur Verfügung stellen. Um Abweichungen vom Produktionsplan festzustellen, gleicht das SCEM des Modeunternehmens diese Daten mit den hinterlegten Lieferterminen ab, indem es auf die Produktionsauftragsdaten (Liefertermin, Liefermenge, etc.) im ERPSystem zugreift. Entsprechende Services können durch das ERP-System des Modeunternehmens bereitgestellt werden.

Melden: Bei Feststellung einer Produktionsverzögerung sind die jeweiligen Prozessverantwortlichen zu benachrichtigen (bspw. per E-Mail oder SMS). Zur Bereitstellung korrespondierender Services kommen neben dem Modeunternehmen selbst auch Webserviceprovider wie ,StrikeIron“ infrage. Zur Gewährleistung eines Eskalationsmechanismus kann das Modeunternehmen über Funktionskapselung in eigenen Systemen (Human Resource Management, Active Directory, etc.) den Anwesenheitsstatus der Prozessverantwortlichen, deren Stellvertreter sowie die zugehörigen Kontaktdaten abfragen.

Simulieren: Um den Prozessverantwortlichen die Auswirkungen der Produktionsverzögerung zu verdeutlichen (insbesondere, ob ein vereinbarter Liefertermin gefährdet wird), reicht bspw. bereits ein lokaler Service, der Prozessdurchlaufzeiten (aus einem Data 


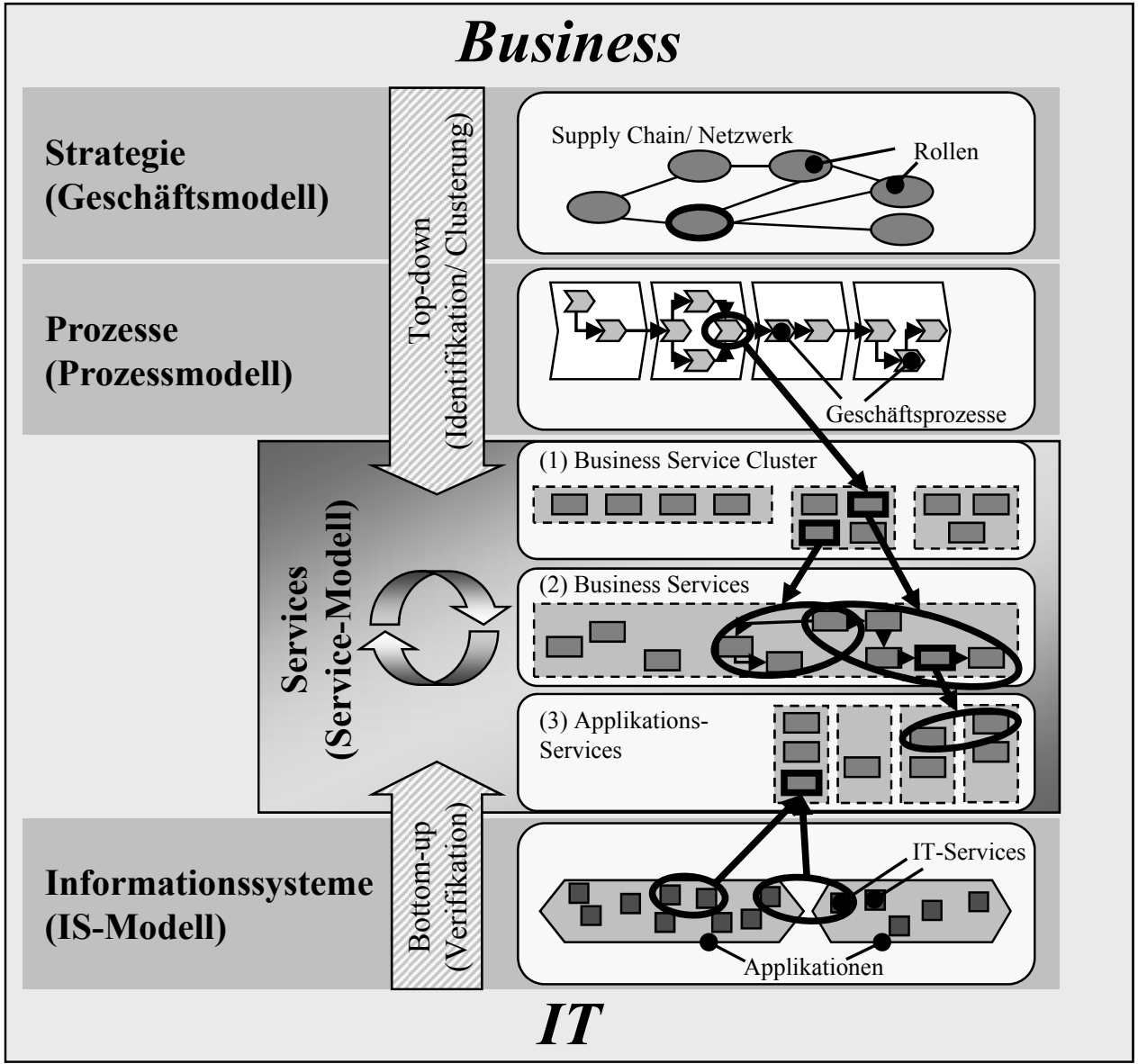

Abbildung 3: Service-Identifizierungs-Framework (in Anlehnung an Österle 1995, 28; Kohlmann/Alt 2009 a, 205 und 2009 b, 111)

Warehouse o. ä.) abgreift und diese auf die Dauer der festgestellten Verzögerung addiert. Soll das SCEM belastbare Handlungsvorschläge anbieten, sind zu deren Generierung Echtzeitdaten der Wertschöpfungspartner erforderlich, die das Modeunternehmen über Webservices abfragen kann. Hierzu zählen bspw. Anfragen zu freien Kapazitäten (Produzenten, Transport- und Logistikdienstleister) oder Frachttarifauskünfte (Transportdienstleister).

Steuern: Entscheidet sich der Supply Chain Manager für eine der vorgeschlagenen Handlungsalternativen (bspw. einen Wechsel von See- auf Lufttransport, um die durch die Produktionsverzögerung verspätete Charge noch rechtzeitig ausliefern zu können), führt dies - ähnlich wie bei ,Simulieren` - zum Aufruf von Webservices bei den involvierten Ge- 
schäftspartnern, die u. a. bestehende Aufträge modifizieren/stornieren bzw. neue Aufträge platzieren können.

Messen: Zur mittel- bis langfristig Reduzierung von Produktionsverzögerungen speichert ein Archivierungsservice kontinuierlich die relevanten Daten und stellt diese in aufbereiteter Form den Prozessverantwortlichen z. B. durch Services zum automatisierten Extrahieren/Transformieren/Laden (ETL) zur Verfügung. Auf dieser Basis können entsprechende Anschlussmassnahmen mittels der erzeugten Metriken (Anzahl an Produktionsverzögerungen je Produzent/Herstellungsland/Produkttyp/etc.) auf taktischer bzw. strategischer SCM-Ebene abgeleitet werden.

\subsection{Serviceorientierte SCEM-Systemarchitektur für die Modeindustrie}

Auf Basis von identifizierten fachlichen SCEM-Services für die zehn kritischsten Events (vgl. Tröger/Alt 2010) zeigt Abbildung 4 die serviceorientierte Systemarchitektur eines SCEM auf Business Service-Ebene für eine Supply Chain der Modeindustrie aus Sicht eines fokalen Unternehmens. In einem globalen Geschäftsnetzwerk der Modeindustrie fungiert ein solches Unternehmen meist als Value Chain Integrator (Alt 2008, 174, 280), d. h. es trägt die Verantwortung für das rechtzeitige Bereitstellen der entwickelten Produkte in der richtigen Menge am richtigen Ort in der richtigen Qualität und zu den richtigen Kosten.

Der Architekturvorschlag illustriert auch die Verteilung und die Integration der mithilfe des oben vorgestellten Frameworks identifizierten SCEM-relevanten Services (vgl. Abbildung 3). Aus Gründen der Übersichtlichkeit abstrahiert die Architektur dabei von Applikations- und IT-Services. Die Struktur orientiert sich am Grundprinzip der SOA (d. h., die Interaktion zwischen Service-Anbieter, Service-Nachfrager sowie Service-Verzeichnis bzw. -Repository) und beinhaltet die drei in Abschnitt 1.3 eingeführten SOA-Elemente Service, Service-Verzeichnis bzw. -Repository und ESB.

Dargestellt sind zudem die Rollen in einem typischen Geschäftsnetzwerk der Modeindustrie (d. h. ausser dem Modeunternehmen selbst dessen Kunden, Logistikdienstleister, Transportdienstleister, Produzenten, Lieferanten (bspw. für Stoffe/Zutaten) sowie sonstige, nicht unmittelbar zur Modeindustrie zählende Service Provider). Diese Verteilung geht von einem dezentralen Ansatz aus, worin - im Idealfall - jeder Wertschöpfungspartner ein Service Repository betreibt, dessen SCEM-relevante (Web-)Services (z. B. T\&T, Abfrage freier Kapazitäten, Frachttarifauskunft, etc.) über einen Verzeichnisdienst veröffentlicht werden, sodass weitere Unternehmen diese auffinden und ggf. in ihre Servicearchitektur einbinden können. Ebenso wäre eine bilaterale Lösung vorstellbar, bei welcher die Partner (des Modeunternehmens) die Services direkt bereitgestellt erhalten. Zahlreiche SCEM-relevante Services (bspw. E-Mail-Benachrichtigung, Abfrage von Lieferterminen, etc.) können Modeunternehmen auch selbst aus der Funktionskapselung eigener, vorhandener Applikationen ableiten.

Mit diesem Konzept, welches die SCEM-inhärenten Potenziale mit den Vorzügen einer SOA kombiniert, haben Lieferketten die Möglichkeit zur Generierung von Wettbewerbsvorteilen: Erstens deckt die Lösung im Gegensatz zu den bisherigen Architekturansätzen alle SCEM-spezifischen Funktionalitäten (vgl. Abschnitte 1.2 und 3.1) ab. Zweitens wirken die SOA-Eigenschaften verstärkend auf die SCEM-Nutzeffekte (siehe Abschnitte 2.1 und 2.2). Drittens können durch die Designprinzipien einer SOA - Standardisierung, lose Kopplung, und Modularität - zusätzliche (zeit-, kosten- und qualitätsbezogene) Nutzeffekte entstehen (vgl. Abschnitt 2.3). 


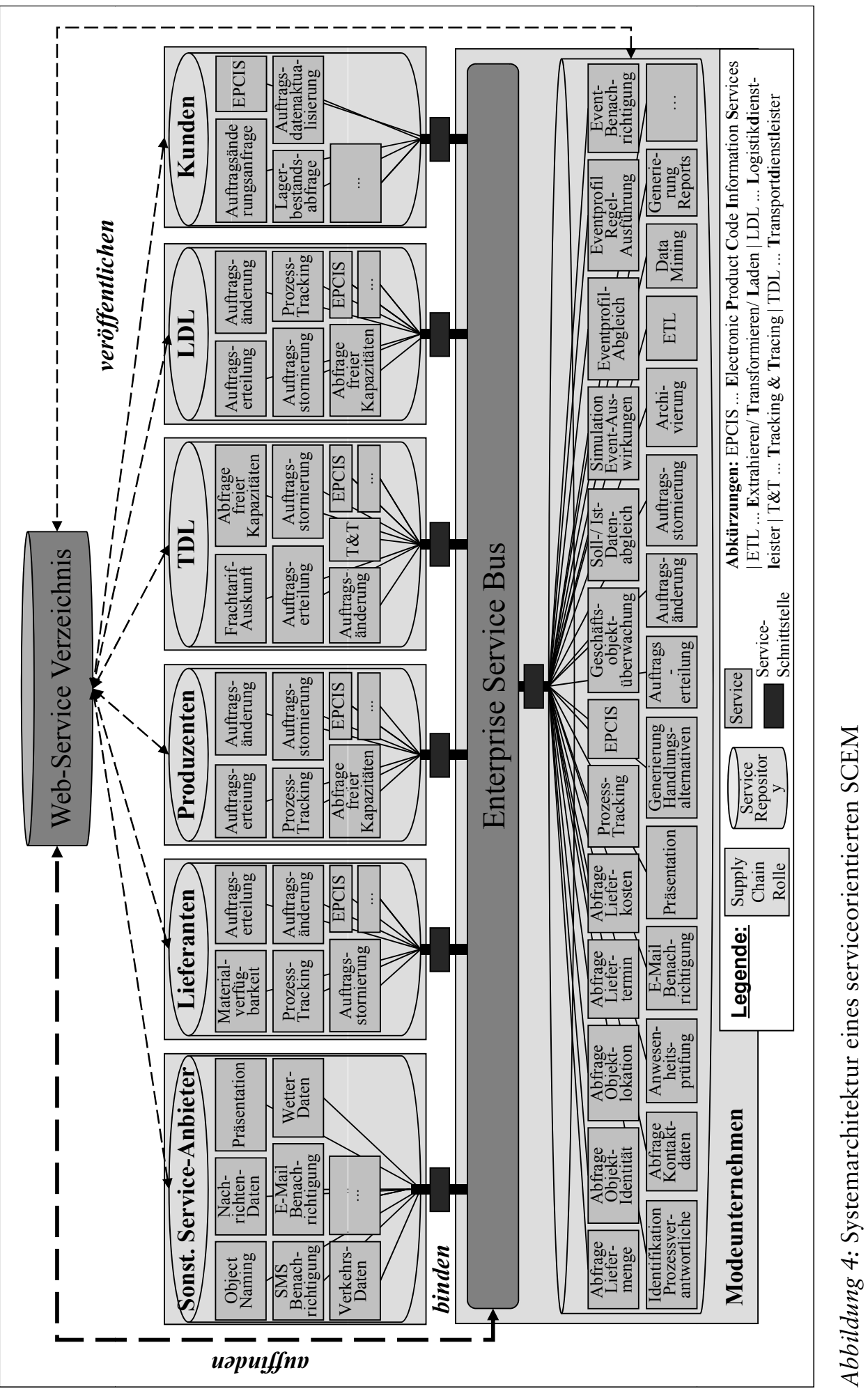




\section{Fazit und Ausblick}

Dieser Beitrag liefert mehrere Ansatzpunkte für das Management globaler Wertschöpfungsketten. Erstens ist die bislang ausstehende Systematisierung der Nutzenpotenziale des SCEM anzuführen. Unter Verwendung dieser Potenziale hat der Beitrag zweitens mögliche Anknüpfungspunkte für den Nutzen von SOA im SCEM identifiziert. Schliesslich umfasst der Beitrag einen Vorschlag für die Ausgestaltung einer serviceorientierten SCEM-Systemarchitektur in der Modeindustrie.

Inhalt und Ergebnisse dieses Beitrages bilden ferner einen Ausgangpunkt für zukünftige Forschungsaktivitäten: bspw. wäre ein Wirtschaftlichkeitsvergleich zwischen einer konventionellen SCEM-Systemarchitektur (d. h. monolithisch mit entsprechend zahlreichen bilateralen Schnittstellen) und dem hier vorgeschlagenen serviceorientierten Konzept aufschlussreich. Weiterhin wären für Dienstanbieter (bspw. Transport- und Logistikdienstleister, kommerzielle SCEM-Anbieter, etc.) die Identifizierung weiterer SCEM-relevanter Services (bspw. aus Sicht anderer Branchen) und deren Potenzial als neues Geschäftsmodell von praktischer Relevanz. In diesem Kontext besteht eine grosse Herausforderung in der Standardisierung der Services in den jeweiligen Anwendungsdomänen.

Zudem wäre auch eine Untersuchung weiterer SCEM-Architekturszenarien (etwa aus Sicht eines Logistik- oder IT-Dienstleisters) lohnenswert. In diesem Zusammenhang sind organisatorische, technische und geschäftsbezogene Fragestellungen im Kontext globaler Supply Chains denkbar. Darüber hinaus liesse sich auch die Komplexität bei der Umsetzung eines serviceorientierten SCEM-Konzeptes (Implementierung und Betrieb der Service Repositories bei allen Lieferkettenteilnehmern, Sicherstellung von Datenschutz/Datensicherheit/Servicequalität, etc.) thematisieren.

Mit wachsender Durchdringung serviceorientierter Architekturen in der betrieblichen Praxis wird die serviceorientierte Gestaltung von SCEM-Systemen eine zunehmend zweckmässige und nutzbringende Option darstellen. Die Erkenntnisse aus den in den folgenden Jahren zu erwartenden Realisierungen werden für Wissenschaftler und Praktiker gleichermassen interessante Anknüpfungspunkte zur weiteren Optimierung globaler Lieferketten bieten.

\section{Literaturhinweise}

Alt, R. (2009): Innovation durch Services Science, in: FAZ Management Compass, Nr. 2, S. 9-11.

Alt, R. (2008): Überbetriebliches Prozessmanagement: Gestaltungsalternativen und Vorgehen am Beispiel integrierter Prozessportale, Berlin.

Bittel, L. (1964): Management by Exception. Systematizing and Simplifying the Managerial Job, New York.

Bodendorf, F./Zimmermann, R. (2005): Proactive Supply-Chain Event Management with Agent Technology, in: International Journal of Electronic Commerce, Jg. 9, Nr. 4, S. 57-89.

Bose, I./Pal, R. (2005): Auto-ID: Managing Anything, Anywhere, Anytime in the Supply Chain, in: Communications of the ACM, Jg. 48, Nr. 8, S. 100-106.

Bretzke, W., et al. (2002): Vom Tracking \& Tracing zum Supply Chain Event Management - aktueller Stand und Trends, KPMG Consulting AG (Hrsg.), S. 1-45.

Bubl, H., et al. (2008): Service Sciences, in: Wirtschaftsinformatik (1), S. 60-65.

Erl, T. (2008): SOA: Principles of Service Design, Upper Saddles River u. a. 
Fisher, M. (1997): What is the right Supply Chain for your Product, in: Harvard Business Review, March/April, Jg. 75, Nr. 2, S. 105-116.

Gaonkar, R./Viswanadham, N. (2004): A conceptual and analytical framework for the management of risk in supply chains, ISB Working Paper, also published in: Proceedings of the International Conference on Robotics and Automation, New Orleans, S. 2699-2704.

Heusler, K., et al. (2006): Supply Chain Event Management. Grundlagen, Funktionen und potenzielle Akteure, in: WiSt, Jg. 35, Nr. 1, S. 19-24.

Ijioui, R., et al. (2007): Auf Überraschungen vorbereitet: Transparenz durch Supply Chain Event Management, in: REFA-Nachrichten, Nr. 2, S. 28-33.

Kohlmann, F./Alt, R. (2009 a): Business-oriented Service Design in Practice - Learnings from a Swiss Universal Bank, in: Proceedings of the $1^{\text {st }}$ International Symposium on Service Sciences (ISSS), Berlin, S. 201-215.

Kohlmann, F./Alt, R. (2009 b): Vernetzung durch Serviceorientierung, in: Alt, R., et al. (Hrsg.): Transformation von Banken, Praxis des In- und Outsourcings auf dem Weg zur Bank 2015, Berlin/Heidelberg, S. 99-128.

Kurbel, K./Schreber, D. (2005): Mobile SCEM. Agentenbasiertes Event-Management in globalen Logistikketten, in: Industriemanagement, Jg. 21, Nr. 5, S. 21-24.

Legner, C. (2009): Do Web Services Foster Specialization? An Analysis of Web Service Directories, in: Tagungsband der 9. Internationalen Tagung Wirtschaftsinformatik, Band 1, Wien, S. 67-76.

Legner, C./Heutschi, R. (2007): SOA Adoption In Practice - Findings From Early SOA Implementations, in: Österle, H., et al. (Hrsg.): Proceedings of the 15th European Conference on Information Systems (ECIS), St. Gallen, S. 1643-1654.

Müller, B., et al. (2010): Understanding the Economic Potential of Service-Oriented Architecture, in: Journal of Management Information Systems, Jg. 26, Nr. 4, S. 145-180.

Müller, J., et al. (2009): Gain in Transparency versus Investment in the EPC Network - Analysis and Results of a Discrete Event Simulation Based on a Case Study in the Fashion Industry, in: Dan, A., et al. (Hrsg.): Service-Oriented Computing - ICSOC ServiceWave 2009 Workshops, Berlin 2010, S. 145-155.

Nissen, V. (2002): Supply Chain Event Management, in: Wirtschaftsinformatik, Jg. 44, Nr. 5, S. 477-480.

Otto, A. (2003): Supply Chain Event Management: Three Perspectives, in: The International Journal of Logistics Management, Jg. 14, Nr. 2, S. 1-13.

Österle, H. (1995): Business Engineering. Prozess- und Systementwicklung, Band 1: Entwurfstechniken, Berlin.

Papazoglou, M./Van den Heuvel, W.-J. (2007): Service-oriented architectures: approaches, technologies and research issues, in: The VLDB Journal, Nr. 3, S. 289-416.

Schulte, C. (2009): Logistik. Wege zur Optimierung der Supply Chain, 5. Auflage, München.

Speyerer, J./Zeller, A. (2005): Managing Supply Networks: Symptom Recognition and Diagnostic Analysis with Web Services, in: Proceedings of the 37th Hawaii International Conference on System Science (HICSS), Koloa, S. 1-10.

Stölzle, W. (2004): Supply Chain Event Management, in: Klaus, P./Krieger, W. (Hrsg.): Gabler Lexikon Logistik, 3. Auflage, Wiesbaden, S. 503-507.

Straube, F., et al. (2007): International Procurement in Emerging Markets - Discovering the drivers of sourcing success, Bremen. 
Tribowski, C., et al. (2009): EPCIS-based Supply Chain Event Management - A Quantitative Comparison of Candidate System Architectures, in: Proceedings of the $3^{\text {rd }}$ International Conference on Complex, Intelligent and Software Intensive Systems (CISIS), Fukuoka, S. 494-499.

Tröger, R./Alt, R. (2010): Service-oriented Supply Chain Event Management - a Case Study from the Fashion Industry, in: Abramowicz, W., et al. (Hrsg.): Informatik 2010 - Business Process and Service Science - Proceedings of ISSS and BPSC, Band 3, Bonn, S. 31-42.

Tröger, R., et al. (2008): Eventmanagement für Ausnahmefälle, in: Dispo, Nr. 8, S. 22-25.

Werner, H. (2008): Supply Chain Management - Grundlagen, Strategien, Instrumente und Controlling, 3. Auflage, Wiesbaden.

Wieser, O./Lauterbach, B. (2001): Supply Chain Event Management mit mySAP SCM (Supply Chain Management), in: HMD, Jg. 38, Nr. 219, S. 65-71.

Ralph Tröger war 2006-2010 als Projektleiter in verschiedenen Abteilungen der Gerry Weber International AG tätig. Seit 2008 ist er zudem externer Doktorand am Institut für Wirtschaftsinformatik der Universität Leipzig. Seit 2011 ist er Produktmanager EPC/RFID bei GS1 Germany. Seine Forschungsschwerpunkte sind die Themengebiete Supply Chain (Event) Management, Logistik und EPC/RFID.

Anschrift: GS1 Germany GmbH, Maarweg 133, D-50825 Köln, Tel.: +49 (0)221/ 94714-243, E-Mail: r.troeger@wifa.uni-leipzig.de

Rainer Alt, Prof. Dr., ist seit 2006 Professor für Wirtschaftsinformatik, insbesondere Anwendungssysteme in Wirtschaft und Verwaltung, am Institut für Wirtschaftsinformatik der Universität Leipzig. Seine Forschung konzentriert sich auf die Unterstützung und Gestaltung überbetrieblicher Prozesse durch Anwendungssysteme im Banken- und Logistikbereich.

Anschrift: Universität Leipzig, Grimmaische Str. 12, D-04109 Leipzig, Tel.: +49 (0)341/ 97-33600, E-Mail: rainer.alt@uni-leipzig.de 